\title{
UNION-PRESERVING TRANSFORMATIONS OF SPACE
}

\author{
EDWARD KASNER AND JOHN DECICCO
}

1. General statement. Sophus Lie studied transformations from lineal-elements into lineal-elements, and also transformations from surface-elements into surface-elements of space. The contact group is obtained by requiring all unions to be converted into unions. Lie's fundamental theorems may be stated as follows. All the contact linealelement transformations form the group of extended point transformations. The contact surface-element transformations which are not merely extended point transformations are defined completely by either a single directrix equation, or a pair of directrix equations. In the first case, a point corresponds to a surface; and in the second case, a point corresponds to a curve. ${ }^{1}$

We extend the preceding results by studying transformations in space from differential curve-elements of order $n:\left(x, y, z, y^{\prime}, z^{\prime}, \cdots\right.$, $\left.y^{(n)}, z^{(n)}\right)$, where $n$ is 2 or more, into lineal-elements $\left(X, Y, Z, Y^{\prime}, Z^{\prime}\right)$. An example of such a transformation arises in the problem of finding the locus of the centers of spherical curvature for an arbitrary space curve. This problem leads to a transformation from curve-elements of third order into lineal-elements. ${ }^{2}$

We determine the general class of union-preserving transformations by means of a directrix equation. Lie has obtained directrix equations only for contact transformations of surface-elements since there are no contact transformations of lineal-elements besides the extended point transformations. For a point-to-surface transformation, Lie's standard directrix equation is of the form $\Omega(X, Y, Z, x, y, z)=0$. For a point-to-curve transformation, there are two standard directrix equations of the forms $\Omega_{1}(X, Y, Z, x, y, z)=0, \Omega_{2}(X, Y, Z, x, y, z)=0$. We find that any general union-preserving transformation from curveelements of order $n$ into lineal-elements is completely determined by our new directrix equation, involving derivatives, $\Omega(X, Y, Z, x, y, z$, $\left.y^{\prime}, z^{\prime}, \cdots, y^{(n-2)}, z^{(n-2)}\right)=0$.

In the final part of our paper, we shall prove that the only available union-preserving transformations (in the whole domain of curve-

Presented to the Society, November 27, 1943; received by the editors August 17, 1943. We have studied the two-dimensional aspects of our new theory in Proc. Nat. Acad. Sci. (1943) and Revista des Matimaticas (1943). This leads to a large extension of the Huygens theory of evolutes and involutes and Lie theory.

${ }^{1}$ Lie-Scheffers, Beruhrungstransformationen.

2 See Bull. Amer. Math. Soc. abstract 49-9-235 by Kasner and DeCicco, $A$ generalized theory of contact transformations. 
elements) are firstly, the group of point transformations, and secondly, the set of union-preserving transformations from curve-elements of order $n$ into lineal-elements, together with the extensions of these two types.

2. The union-preserving transformations. Any transformation from curve-elements of order $n$, where $n$ is 2 or more, into lineal-elements may be defined by equations of the form

$$
\begin{aligned}
& X=X\left(x, y, z, p_{1}, q_{1}, \cdots, p_{n}, q_{n}\right), \\
& Y=Y\left(x, y, z, p_{1}, q_{1}, \cdots, p_{n}, q_{n}\right), \\
& Z=Z\left(x, y, z, p_{1}, q_{1}, \cdots, p_{n}, q_{n}\right), \\
& P=P\left(x, y, z, p_{1}, q_{1}, \cdots, p_{n}, q_{n}\right), \\
& Q=Q\left(x, y, z, p_{1}, q_{1}, \cdots, p_{n}, q_{n}\right),
\end{aligned}
$$

where $p_{m}=y^{(m)}=d^{m} y / d x^{m}$ and $q_{m}=z^{(m)}=d^{m} z / d x^{m}$ for $m=1,2, \cdots, n$.

A series of curve-elements of order $n$ may be defined by $y=y(x)$, $z=z(x), p_{1}=p_{1}(x), q_{1}=q_{1}(x), \cdots, p_{n}=p_{n}(x), q_{n}=q_{n}(x)$. A series is a union if and only if the conditions $d y-p_{1} d x=0, d z-q_{1} d x=0, \cdots$, $d p_{n-1}-p_{n} d x=0, d q_{n-1}-q_{n} d x=0$ are satisfied.

A special type of union is the conical-union of order $n$. This consists of $\infty^{1}$ curve-elements of order $n$ which have in common a fixed curve-element of order $(n-1)$. The equations of any conical-union are $x=x_{0}, y=y_{0}, z=z_{0}, p_{1}=\left(p_{1}\right)_{0}, q_{1}=\left(q_{1}\right)_{0}, \cdots, p_{n-1}=\left(p_{n-1}\right)_{0}, q_{n-1}$ $=\left(q_{n-1}\right)_{0}, q_{n}=F\left(p_{n}\right)$.

Let us now consider what unions become unions under the transformation (1). These unions must satisfy the pair of Monge differential equations of order $(n+1)$

$$
\begin{aligned}
\left(Y_{p_{n}}\right. & \left.-P X_{p_{n}}\right) p_{n+1}+\left(Y_{q_{n}}-P X_{q_{n}}\right) q_{n+1} \\
\quad & {\left[\left(Y_{x}+p_{1} Y_{y}+q_{1} Y_{z}+\cdots+p_{n} Y_{p_{n-1}}+q_{n} Y_{q_{n-1}}\right)\right.} \\
& \left.-P\left(X_{x}+p_{1} X_{y}+q_{1} X_{z}+\cdots+p_{n} X_{p_{n-1}}+q_{n} X_{q_{n-1}}\right)\right]=0 \\
\left(Z_{p_{n}}\right. & \left.-Q X_{p_{n}}\right) p_{n+1}+\left(Z_{q_{n}}-Q X_{q_{n}}\right) q_{n+1} \\
& +\left[\left(Z_{x}+p_{1} Z_{y}+q_{1} Z_{z}+\cdots+p_{n} Z_{p_{n-1}}+q_{n} Z_{q_{n-1}}\right)\right. \\
& \left.-Q\left(X_{x}+p_{1} X_{y}+q_{1} X_{z}+\cdots+p_{n} X_{p_{n-1}}+q_{n} X_{q_{n-1}}\right)\right]=0 .
\end{aligned}
$$

THEOREM 1. All transformations from curve-elements of order $n$ into lineal-elements may be divided into three distinct types with respect to the number of unions which are converted into unions. ${ }^{3}$

\footnotetext{
${ }^{3}$ Kasner, General transformation theory of differential elements, Amer. J. Math. vol. 32 (1904) pp. 392-401.
} 
Type $1^{\circ}$. The union-preserving transformations. These carry every union into a union. The necessary and sufficient conditions for a unionpreserving transformation are

$$
\begin{aligned}
P & =\frac{Y_{x}+p_{1} Y_{y}+q_{1} Y_{z}+\cdots+p_{n} Y_{p_{n-1}}+q_{n} Y_{q_{n-1}}}{X_{x}+p_{1} X_{y}+q_{1} X_{z}+\cdots+p_{n} X_{p_{n-1}}+q_{n} X_{q_{n-1}}} \\
& =\frac{Y_{p_{n}}}{X_{p_{n}}}=\frac{Y_{q_{n}}}{X_{q_{n}}}, \\
Q & =\frac{Z_{x}+p_{1} Z_{y}+q_{1} Z_{z}+\cdots+p_{n} Z_{p_{n-1}}+q_{n} Z_{q_{n-1}}}{X_{x}+p_{1} X_{y}+q_{1} X_{z}+\cdots+p_{n} X_{p_{n-1}}+q_{n} X_{q_{n-}}} \\
& =\frac{Z_{p_{n}}}{X_{p_{n}}}=\frac{Z_{q_{n}}}{X_{q_{n}}} .
\end{aligned}
$$

Type $2^{\circ}$. The intermediate transformations. These are not unionpreserving transformations and satisfy the conditions

$$
\begin{gathered}
{\left[\left(Y_{x}+p_{1} Y_{y}+q_{1} Y_{z}+\cdots+p_{n} Y_{p_{n-1}}+q_{n} Y_{q_{n-1}}\right)\right.} \\
\left.-P\left(X_{x}+p_{1} X_{y}+q_{1} X_{z}+\cdots+p_{n} X_{p_{n-1}}+q_{n} X_{q_{n-1}}\right)\right] \\
\cdot\left[\left(Z_{x}+p_{1} Z_{y}+q_{1} Z_{z}+\cdots+p_{n} Z_{p_{n-1}}+q_{n} Z_{q_{n-1}}\right)\right. \\
\left.-Q\left(X_{x}+p_{1} X_{y}+q_{1} X_{z}+\cdots+p_{n} X_{p_{n-1}}+q_{n} X_{q_{n-1}}\right)\right]^{-1} \\
=\frac{Y_{p_{n}}-P X_{p_{n}}}{Z_{p_{n}}-Q X_{p_{n}}}=\frac{Y_{q_{n}}-P X_{q_{n}}}{Z_{q_{n}}-Q X_{q_{n}}} .
\end{gathered}
$$

Any intermediate transformation carries the $\infty^{\infty}$ unions of a Monge differential equation of third order into unions.

Type $3^{\circ}$. The general transformations. These do not satisfy the conditions (4). Any such transformation converts precisely $\infty^{2(n+1)}$ unions into unions.

The general Type $3^{\circ}$ is defined by five arbitrary functions of $(2 n+3)$ independent variables. The intermediate Type $2^{\circ}$ is defined by three arbitrary functions of $(2 n+3)$ independent variables. For this intermediate type, it is seen that $(X, Y, Z)$ may be taken arbitrarily and then the functions $P$ and $Q$ are completely determined by the equations (4). Later we shall show that the union-preserving Type $1^{\circ}$ is defined by one arbitrary function of $(2 n+1)$ variables or by three arbitrary functions of $(2 n+1)$ variables.

We prove Theorem 1. For union-preserving transformations the equations (2) must be identities in $p_{n+1}$ and $q_{n+1}$. Upon setting the various coefficients equal to zero and solving for $P$ and $Q$, we obtain 
the conditions (3) for union-preserving transformations.

Next suppose we have the intermediate transformations so that the conditions (4) are satisfied. Then it is obvious that the equations (2) are equivalent. This shows that under any intermediate transformation, there are $\infty^{\infty}$ unions preserved. These satisfy either one of the two equivalent Monge differential equations (2).

Finally suppose the conditions (4) are not satisfied. In the first place it is seen that if the last equality of (4) is not valid, we can solve the equations (2) for $p_{n+1}$ and $q_{n+1}$. Therefore there are $\infty^{2(n+1)}$ unions which become unions. Finally if the last equality of (4) is valid, then it can be shown that at each curve-element of order $(n-1)$ there are $\infty^{1}$ conical-unions which become unions. Since there are $\infty^{(2 n+1)}$ curve-elements of order $(n-1)$, it follows that altogether there are $\infty^{2(n+1)}$ conical-unions which are converted into unions. Therefore we have proved that our general Type $3^{\circ}$ carries exactly $\infty^{2(n+1)}$ unions into unions under all conditions.

3. The degenerate union-preserving transformations. Henceforth we shall consider only union-preserving transformations. However, there are certain degenerate union-preserving transformations which we wish to exclude from consideration. These degenerate correspondences are those where $P$ and $Q$ assume identically the values 0 or $\infty$; and also those for which there exist two functional relations between the three functions definining $(X, Y, Z)$.

It is found that these degenerate transformations may be divided into four cases: (a) those which convert every union into a union in a fixed plane parallel to one of the coordinate planes, (b) those which carry every union into a fixed straight line parallel to one of the coordinate axes, (c) those which carry every union into a conical-union of lineal-elements with fixed base-point, and (d) those which carry every union into a single fixed union.

In the rest of our article, whenever we speak of a union-preserving transformation we shall mean one which is not of the four preceding degenerate types.

4. The special union-preserving transformations. Any (nondegenerate) union-preserving transformation is said to be special if it carries every conical-union of order $n$ into a conical-union of lineal-elements. Any such special transformation is given by equations of the form

$$
\begin{aligned}
& X=X\left(x, y, z, p_{1}, q_{1}, \cdots, p_{n-1}, q_{n-1}\right), \\
& Y=Y\left(x, y, z, p_{1}, q_{1}, \cdots, p_{n-1}, q_{n-1}\right), \\
& Z=Z\left(x, y, z, p_{1}, q_{1}, \cdots, p_{n-1}, q_{n-1}\right),
\end{aligned}
$$




$$
\begin{aligned}
& P=\frac{Y_{x}+p_{1} Y_{y}+q_{1} Y_{z}+\cdots+p_{n} Y_{p_{n-1}}+q_{n} Y_{q_{n-1}}}{X_{x}+p_{1} X_{y}+q_{1} X_{z}+\cdots+p_{n} X_{p_{n-1}}+q_{n} X_{q_{n-1}}} \\
& Q=\frac{Z_{x}+p_{1} Z_{y}+q_{1} Z_{z}+\cdots+p_{n} Z_{p_{n-1}}+q_{n} Z_{q_{n-1}}}{X_{x}+p_{1} X_{y}+q_{1} X_{z}+\cdots+p_{n} X_{p_{n-1}}+q_{n} X_{q_{n-1}}} .
\end{aligned}
$$

These special union-preserving transformations depend on three arbitrary functions of $(2 n+1)$ independent variables. Thus there are $\infty^{3 f(2 n+1)}$ special union-preserving transformations.

5. The directrix equation of a general union-preserving transformation $T$. We shall say that a union-preserving transformation $T$ is general if it does not carry every conical-union of order $n$ into a conical-union of lineal-elements. It follows that for such a general transformation $T$, we must have either $X_{p_{n}} \neq 0, Y_{p_{n}} \neq 0, Z_{p_{n}} \neq 0$, or $X_{q_{n}} \neq 0$, $Y_{q_{n}} \neq 0, Z_{q_{n}} \neq 0$, or both.

THEOREM 2. For a general union-preserving transformation $T$, the four variables $\left(p_{n-1}, q_{n-1}, p_{n}, q_{n}\right)$ can be eliminated from the three functions defining $(X, Y, Z)$, thus obtaining a single eliminant of the form

$$
\Omega\left(X, Y, Z, x, y, z, p_{1}, q_{1}, \cdots, p_{n-2}, q_{n-2}\right)=0 .
$$

We call this the directrix equation of our general union-preserving transformation $T$.

Let us proceed with the proof of the above result. In the first place, it is seen by (3) that $p_{n}$ and $q_{n}$ can be eliminated from the first and second, and also from the first and third of our equations (1) defining the general union-preserving transformation $T$. We therefore obtain the two relations

$$
\begin{aligned}
& Y=F\left(X, x, y, z, p_{1}, q_{1}, \cdots, p_{n-1}, q_{n-1}\right), \\
& Z=G\left(X, x, y, z, p_{1}, q_{1}, \cdots, p_{n-1}, q_{n-1}\right) .
\end{aligned}
$$

At this point, we wish to introduce the following notation. Let $\mathrm{X}$ be any function of $\left(X, Y, Z, x, y, z, p_{1}, q_{1}, \cdots, p_{m}, q_{m}\right)$. Then the operation prime on $\mathrm{X}$ denotes the following linear differential operator

$$
\mathrm{X}^{\prime}=\mathrm{X}_{x}+p_{1} \mathrm{X}_{y}+q_{1} \mathrm{X}_{z}+\cdots+p_{m+1} \mathbf{X}_{p_{m}}+q_{m+1} \mathbf{X}_{\dot{q}_{m}} \text {. }
$$

That is, $\mathrm{X}^{\prime}$ denotes the total derivative of $\mathrm{X}$ with respect to $x$ while considering $(X, Y, Z)$ as constants.

Next upon applying the conditions (3) for a union-preserving transformation, we discover that

$$
P=F_{X}, \quad Q=G_{X}, \quad F^{\prime}=0, \quad G^{\prime}=0 .
$$


The eliminant with respect to $X$ of the last two of the preceding equations must be identically zero. Differentiating the last two equations with respect to $p_{n}$, and also with respect to $q_{n}$, we find

$$
\begin{array}{ll}
X_{p_{n}} F_{X}^{\prime}+F_{p_{n-1}}=0, & X_{q_{n}} F_{X}^{\prime}+F_{q_{n-1}}=0, \\
X_{p_{n}} G_{X}^{\prime}+G_{p_{n-1}}=0, & X_{q_{n}} G_{X}^{\prime}+G_{q_{n-1}}=0 .
\end{array}
$$

From these four equations we can eliminate $F_{X^{\prime}}$ and $G_{X^{\prime}}$, thus finding the two eliminants

$$
X_{q_{n}} F_{p_{n-1}}-X_{p_{n}} F_{q_{n-1}}=0, \quad X_{q_{n}} G_{p_{n-1}}-X_{p_{n}} G_{q_{n-1}}=0 .
$$

Since $X_{p_{n}}$ and $X_{q_{n}}$ can not both be zero, it follows from these equations that

$$
F_{p_{n-1}} G_{q_{n-1}}-F_{q_{n-1}} G_{p_{n-1}}=0 .
$$

Observe that both $F$ and $G$ are not independent of $p_{n-1}$ and $q_{n-1}$. For otherwise, if $X$ is explicitly present in either $F^{\prime}$ or $G^{\prime}, X$ would be independent of $p_{n}$ and $q_{n}$ as follows from equations (9). Hence $X$ is not present in the last two of equations (9), and it results that both $F$ and $G$ are functions of $X$ only. This is impossible since our transformation is not degenerate.

By the preceding remarks and equation (12), it follows that (7) has one and only one eliminant with respect to $p_{n-1}$ and $q_{n-1}$, namely the equation (6). This completes the proof of Theorem 2.

THEOREM 3. The directrix equation (6) completely determines the general union-preserving transformation $T$, and conversely.

The general union-preserving transformations depend upon one arbitrary function of $(2 n+1)$ independent variables. Thus there are $\infty f(2 n+1)$ general union-preserving transformations.

In the first place, it is seen that under the general union-preserving transformation $T$ any point in the $(X, Y, Z)$-space corresponds to a family of $\infty^{\infty}$ curves in the $(x, y, z)$-space, defined by a Monge equation of order $(n-2)$. These curves are defined by (6), where $(X, Y, Z)$ are considered as constants. The derivatives of orders $(n-2),(n-1)$, $n$ of these curves are given by the equations

$$
\Omega=0, \quad \Omega^{\prime}=0, \quad \Omega^{\prime \prime}=0 .
$$

Next any conical-union of order $n$ in the $(x, y, z)$-space is converted into a union. This union is defined by the first two of the preceding equations where $\left(x, y, z, p_{1}, q_{1}, \cdots, p_{n-1}, q_{n-1}\right)$ are regarded as constants. The first derivatives of this union are given by the equations 


$$
\Omega_{X}+P \Omega_{Y}+Q \Omega_{Z}=0, \quad \Omega_{X}^{\prime}+P \Omega_{Y}^{\prime}+Q \Omega_{Z}^{\prime}=0 .
$$

We shall prove that the functions defining $(X, Y, Z, P, Q)$ of our general union-preserving transformation $T$ must satisfy the equations (13) and (14). In the first place $(X, Y, Z)$ must satisfy the directrix equation (6), since it was obtained as a result of eliminating $\left(p_{n-1}, q_{n-1}, p_{n}, q_{n}\right)$ from the first three of equations (1) defining our general union-preserving transformation $T$.

Applying the conditions (3) for union-preserving transformations to our directrix equation (6), we obtain the first two of equations (13) and the first of equations (14).

Finally upon applying the conditions (3) for union-preserving transformations to the second of equations (13), we obtain the last of equations (13) and also the last of equations (14).

That we can actually solve equations (13) and (14) for ( $X, Y, Z$, $P, Q$ ) follows from the fact that the directrix equation (6) represents a three-parameter family of Monge equations of order $(n-2)$ in the $(x, y, z)$-space (or a Monge equation of order $(n+1)$ obtained by eliminating the parameters $(X, Y, Z)$, which may be integrated three times).

By Theorems 2 and 3, it follows that the transformation from curve-elements of third order into lineal-elements, which is obtained from the problem of finding the locus of the center of spherical curvature to an arbitrary curve, is general union-preserving. On the other hand, the problem of finding the locus of the center of circular curvature to an arbitrary curve leads to a transformation from curveelements of third-order into lineal-elements which is special unionpreserving.

We note the analogy to Lie's theory of contact transformations of surface-elements. In his work, either a transformation is point-topoint, or else it is point-to-surface or point-to-curve, in which cases it is defined by a single directrix equation or by a pair of directrix equations. In our theory, a union-preserving transformation is special or general, in which case it is defined by a single directrix equation.

\section{The union-preserving transformations of space in the domain of} curve-elements. We shall now discuss the following result. ${ }^{4}$

${ }^{4}$ Kasner and DeCicco, Transformation theory of integrable double-series of linealelements, Bull. Amer. Math. Soc. vol. 46 (1940) pp. 93-100. Kasner, Lineal element transformations of space for which normal congruences of curves are converted into normal congruences, Duke Math. J. vol. 5 (1939) pp. 72-83. Kasner and DeCicco, Curvature element transformations which preserve integrable fields, Proc. Nat. Acad. Sci. U.S.A. vol. 25 (1939) pp. 104-111. 
THEOREM 4. If a union-preserving transformation $T$ from curveelements into lineal-elements carries any two unions which possess $n \geqq 2$ as the order of contact into two unions which have, at least, second order contact, then $T$ must be an extended point-transformation.

In the first place, it is obvious that our union-preserving transformation $T$ is at most from curve-elements of order $n$ into linealelements.

Let us first of all assume that $T$ is a general union-preserving transformation. The first derivatives $P=d Y / d X$ and $Q=d Z / d X$ of any transformed union under the general union-preserving transformation $T$ defined by (13) and (14) are given by (14). We proceed to find the second derivatives $d P / d X=d^{2} Y / d X^{2}$ and $d Q / d X=d^{2} Z / d X^{2}$.

In the first place, by differentiating the last of equations (13) totally with respect to $x$, we find

$$
\left(\Omega_{X}^{\prime \prime}+P \Omega_{Y}^{\prime \prime}+Q \Omega_{Z}^{\prime \prime}\right) d X / d x=-\Omega^{\prime \prime \prime} .
$$

Next differentiating the equations (14) totally with respect to $x$ and using the conditions (14) and (15), we find

$$
\begin{aligned}
& \Omega_{Y} d P / d X+\Omega_{Z} d Q / d X+\Omega_{X X}+P^{2} \Omega_{Y Y}+Q^{2} \Omega_{Z Z} \\
& \quad+2 P \Omega_{X Y}+2 Q \Omega_{X Z}+2 P Q \Omega_{Y Z}=0 \\
& \Omega_{Y}^{\prime} d P / d X+\Omega_{Z}^{\prime} d Q / d X+\Omega_{X X}^{\prime}+P^{2} \Omega_{Y Y}^{\prime}+Q^{2} \Omega_{Z Z}^{\prime} \\
& \quad+2 P \Omega_{X Y}^{\prime}+2 Q \Omega_{X Z}^{\prime}+2 P Q \Omega_{Y Z}^{\prime}=\left(1 / \Omega^{\prime \prime \prime}\right)\left(\Omega_{X}^{\prime \prime}+P \Omega_{Y}^{\prime \prime}+Q \Omega_{Z}^{\prime \prime}\right)^{2} .
\end{aligned}
$$

Now apply the conditions of our theorem. Then $d P / d X$ and $d Q / d X$ can not contain $p_{n+1}$ and $q_{n+1}$. The only place where these appear are in $\Omega^{\prime \prime \prime}$. Their coefficients are $\Omega_{p_{n-2}}$ and $\Omega_{q_{n-2}}$ which can not be zero. Therefore our transformation $T$ is not a general union-preserving transformation from curve-elements of order $n$ into lineal-elements.

Finally let us suppose that our transformation $T$ is special unionpreserving from curve-elements of order $n$ into lineal-elements. By (5), it is found upon extending our transformation $T$ once and imposing the condition that $d P / d X$ and $d Q / d X$ be independent of $p_{n+1}$ and $q_{n+1}$ that our special union-transformation $T$ is a union-preserving transformation from curve-elements of order $(n-1)$, at most, into lineal-elements. This is impossible.

The preceding two contradictions lead us to the conclusion that there are no union-preserving transformations from curve-elements of order $n$ into lineal-elements, such that it converts any two unions 
which possess $n \geqq 2$ as the order of contact into two unions which have at least second order contact.

Therefore the only possible union-preserving transformations which satisfy the conditions of our theorem are those from linealelements into lineal-elements. According to Lie's theorem, it follows that the only such transformations are the extended point transformations. This completes the proof of Theorem 4 .

From this theorem, it follows that the only union-preserving transformations from curve-elements of order $n$ into curve-elements of order $m$ where $n \geqq m>1$ are the extended point transformations. Therefore the only successful union-preserving transformations (in the whole domain of curve-elements) are firstly, the point transformations, and secondly, the union-preserving transformations from curve-elements of order $n$, where $n$ is 2 or more, into lineal-elements, or the extensions of these two types.

Thus if $T$ is a union-preserving transformation from curve-elements of order $n$ into curve-elements of order $m$ where $n \geqq m \geqq 1$, then, if $n=m, T$ is the extension of order $m$ of a point-transformation; and if $n>m, T$ is the extension of order $(m-1)$ of a union-preserving transformation from curve-elements of order $(n-m+1)$ into linealelements.

7. Alternate characterization of union-preserving transformations. Under a union-preserving transformation $T$, a single union in the $(x, y, z)$-space corresponds to a single union in the $(X, Y, Z)$-space, but to a single union in the $(X, Y, Z)$-space there correspond $\infty^{2(n-1)}$ unions in the $(x, y, z)$-space.

(It is noted that a point $\left(X_{0}, Y_{0}, Z_{0}\right)$ is not considered to be a union of lineal-elements in the $(X, Y, Z)$-space, but consists of $\infty f(1)$ conical-unions of lineal-elements, each such conical-union corresponding to $\infty^{2(n-1)}$ unions of the Monge equation of order $(n-2)$, defined by our directrix equation (6)).

TheOREM 5. Any transformation from curve-elements of order $n$ of the $(x, y, z)$-space into lineal-elements of the $(X, Y, Z)$-space, by which there correspond exactly $\infty^{2(n-1)}$ unions in the $(x, y, z)$-space to any single union of the $(X, Y, Z)$-space, is a union-preserving transformation.

Let $Y=F(x), Z=G(x), P=F_{X}, Q=G_{X}$ be any union in the ( $X, Y$, $Z)$-space. Upon applying our transformation (1) to this union, we find that the corresponding unions in the $(x, y, z)$-space must satisfy the differential equations 


$$
\begin{aligned}
& Y\left(x, y, z, p_{1}, q_{1}, \cdots, p_{n}, q_{n}\right)=F\left[X\left(x, y, z, p_{1}, q_{1}, \cdots, p_{n}, q_{n}\right)\right], \\
& Z\left(x, y, z, p_{1}, q_{1}, \cdots, p_{n}, q_{n}\right)=G\left[X\left(x, y, z, p_{1}, q_{1}, \cdots, p_{n}, q_{n}\right)\right], \\
& P\left(x, y, z, p_{1}, q_{1}, \cdots, p_{n}, q_{n}\right)=F_{X}, Q\left(x, y, z, p_{1}, q_{1}, \cdots, p_{n}, q_{n}\right)=G_{X} .
\end{aligned}
$$

In general, we can solve these equations for $\left(p_{n-1}, q_{n-1}, p_{n}, q_{n}\right)$ in terms of $\left(x, y, z, p_{1}, q_{1}, \cdots, p_{n-2}, q_{n-2}\right)$. Thus we find that there are at most $\infty^{2(n-1)}$ unions in the $(x, y, z)$-space which correspond to a given union in the $(X, Y, Z)$-space.

We now impose the conditions that these represent exactly $\infty^{2(n-1)}$ unions. In the preceding equations, we can think of $\left(p_{n-1}, q_{n-1}, p_{n}, q_{n}\right)$ as being functions of $\left(x, y, z, p_{1}, q_{1}, \cdots, p_{n-2}, q_{n-2}\right)$. We can differentiate the first equation partially with respect to $\left(x, y, z, p_{1}, q_{1}, \cdots\right.$, $\left.p_{n-2}, q_{n-2}\right)$, obtaining a set of $(2 n-1)$ partial equations. Multiplying these equations by $\left(1, p_{1}, q_{1}, \cdots, p_{n-1}, q_{n-1}\right)$ respectively, adding the results, and using the conditions that $p_{n}$ is the derivative of $p_{n-1}$ and $q_{n}$ is the derivative of $q_{n-1}$, we obtain an equation of the same form as the first of equations (2). Similarly operating upon the second of the preceding equations, we are led to an equation of the same form as the second of equations (2). From these equations of the form (2), we are led to the conclusion that our transformation is union-preserving since the equations must be independent of $p_{n+1}$ and $q_{n+1}$. This completes the proof of Theorem 5 .

As an application, consider the osculating sphere to any arbitrary curve. To a single curve there corresponds a single spherical evolute, that is, the locus of the centers of spherical curvature. But to a given spherical evolute there correspond $\infty^{4}$ spherical involutes. Thus our work may be considered to be a generalized theory of spherical evolutes and involutes in space.

Columbia UNIVERsity AND

ILLINOIS INSTITUTE OF TECHNOLOGY 